\title{
IMPLEMENTATION OF DIFFERENTIAL EVOLUTION ALGORITHM TO PERFORM IMAGE FUSION FOR IDENTIFYING BRAIN TUMOR
}

\author{
Pothiraj Sivakumar \\ Department of Electronics and Communication Engineering, \\ Kalasalingam Academy of Research and Education, \\ Virudhunagar District, Tamil Nadu, (India). \\ E-mail: siva@klu.ac.in ORCID: https://orcid.org/0000-0003-1328-8093
}

Subbiah Parvathy Velmurugan

Department of Electronics and Communication Engineering,

Kalasalingam Academy of Research and Education,

Virudhunagar District, Tamil Nadu, (India).

E-mail: s.p.velmurugan@klu.ac.in ORCID: https://orcid.org/0000-0002-3314-1454

Jenyfal Sampson

Department of Electronics and Communication Engineering, Kalasalingam Academy of Research and Education,

Virudhunagar District, Tamil Nadu, (India). E-mail: jenyfal.sampson@klu.ac.in ORCID: https://orcid.org/0000-0001-8007-3995

Recepción: 05/12/2019 Aceptación: 30/12/2019 Publicación: 23/03/2020

\section{Citación sugerida:}

Sivakumar, P., Velmurugan, S. P., y Sampson, J. (2020). Implementation of differential evolution algorithm to perform image fusion for identifying brain tumor. 3C Tecnología. Glosas de innovación aplicadas a la pyme. Edición Especial, Marzo 2020, 301-311. http://doi.org/10.17993/3ctecno.2020. specialissue4.301-311

\section{Suggested citation:}

Sivakumar, P., Velmurugan, S. P., \& Sampson, J. (2020). Implementation of differential evolution algorithm to perform image fusion for identifying brain tumor. 3C Tecnología. Glosas de innovación aplicadas a la pyme. Edición Especial, Marzo 2020, 301-311. http://doi.org/10.17993/3ctecno.2020. specialissue4.301-311 


\section{ABSTRACT}

Automated mechanization for curing a disease is a reliable and protuberant method. A disease in brain can be detected by Magnetic Resonance Imaging (MRI). In this context, image fusion is a method for creating an image by merging pertinent data from 2 or more images. The resultant image will be highly useful than the individual input images to retentive the vital characteristics of every image. Multiple image fusion is a significant method employed in image processing techniques. In this study, differential evolution (DE) algorithm-based image fusion has been performed with MRI and computed tomography (CT) images. The simulation works have been carried out to evaluate the different quality measurements of DE on image fusion.

\section{KEYWORDS}

De-speckling, Brain tumor detection, CT, DE, Image fusion, MRI. 


\section{INTRODUCTION}

Brain tumours is harmful to humans, due to the atypical availability of cells inside the brain. Brain function will be interrupted and be deadly. Benign and malignant tumors are frequently identified. Benign tumors are not as harmful as malignant tumors, because they can grow rapidly. Medical imaging methodologies such as MRI, CT, Ultrasound, $\mathrm{X}$-ray etc. are employed to display the internal body parts for diagnosing (Rowden, 2019). Among them MRI is widely employed and it offers accurate brain images and cancer cells. So, brain tumor can be detected via MRI images. This study concentrates on detection of brain tumor through image fusion. Image fusion is a process of merging two or more images into a single compound image that contains the information of the source images without clamor. Multi-modular recuperative image fusion has been employed to recognize the wounds. In biomedical image processing image fusion has got more attention in the past decade (Daneshvar \& Ghassemian, 2010; Wang, Li, \& Tian, 2014). MRI and CT images held more practical information than biomedical images. The aim of image fusion is to obtain the information at each pixel without damaging the pixel associations of the particular image.

In this context, previously, a complex wavelet modification for image fusion has been proposed to attain the optimal combination using Lifting wavelet transform (LWT), Multiwavelet transform (MWT), Stationary wavelet transform (SWT) and spatial domain (Principal component analysis (PCA) approaches (Singh \& Khare, 2014). Similarly, undecimated wavelet has been implemented, where the image is crumbled into two successive scrutinizing errands (Ellmauthaler et al., 2013). An affable fusion technique using SWT and NSCT has been presented, where the input image is rotten by SWT and NSCT. The coefficients of SWT and NSGT are combined to form the fused image (Li \& Liu, 2009). A new framework has been proposed where the images considered with SWT primarily and the overall textural topographies have been attained via gray level cooccurrence matrix (Singh \& Khare, 2014; Huang et al., 2014; Shi \& Fang, 2007). Hence, a scheme for fusing MRI and CT images using DE based Debauchee's wavelet Transform (DE-DWT) has been attempted in this study. 


\section{MATERIALS AND METHODS}

As a part of image fusion, pre-handling of images have been performed using DE. DE has been employed to create the fission rubrics. The preprocessing steps involved in image fusion have been illustrated in Figure 1.

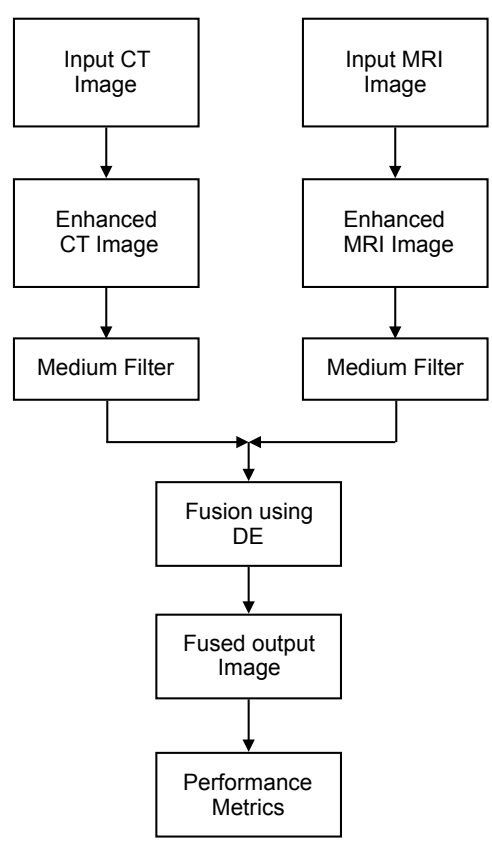

Figure 1. Flowchart of proposed approach.

Primarily, the informative source images such as CT and MRI images have been collected. Subsequently, the source images have been converted into dark scale and resized. The enhancement of quality of the images has been performed using imadjust order available in MATLAB simulation. Commotion dismissal has been carried out by using median channel. This is an excellent method in ejecting salt and pepper commotions of biomedical images. It happens due to the movement of antiquities.

Performance indices such as Mean Square Error (MSE), Peak Signal to Noise Ratio (PSNR), contrast and homogeneity have been estimated. The amount of clamor available in the image is denoted as PSNR. It is used to indicate the obtained fused image has tumbleddown or not. MSE value need to be low and PSNR value need to be high. 


$$
\begin{gathered}
M S E=\frac{1}{m n} \sum_{i=0}^{m-1} \sum_{j=0}^{n-1}[(i, j)-K(i, j)]^{2} \\
P S N R=20 \log _{10}\left(\frac{M A X}{\sqrt{M S E}}\right)
\end{gathered}
$$

Contrast reinstates the data associated with the pixel with the adjacent pixel. It has been calculated as follows.

$$
\text { Contrast }=\sum(i-j) 2 \times P(i, j)
$$

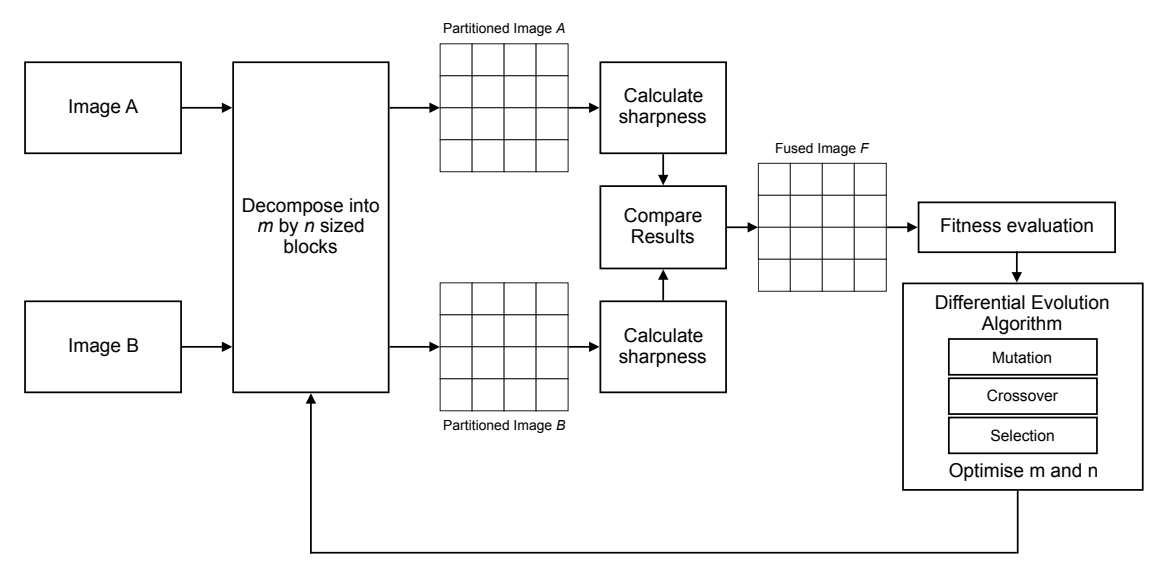

Figure 2. Flowchart for image fusion using DE.

Table 1. Best parameters of DE.

\begin{tabular}{|c|c|}
\hline Parameter & Value \\
\hline Number of population & 100 \\
\hline Maximum generation & 100 \\
\hline Crossover probability & 0.5 \\
\hline Scaling factor & 0.9 \\
\hline
\end{tabular}

Homogeneity has been used to estimate the intimacy of components availed in gray level concurrence matrix (GLCM).

\section{DIFFERENTIAL EVOLUTION ALGORITHM}

Price and Storn introduced DE as a population-based stochastic direct search technique. The implementation procedure of DE has been adopted from Aslantas and Toprak (2014). 
The steps involved in DE based image fusion have been illustrated in Figure 2. The best control parameters for DE have been provided in Table 1 .

The performance indices such as MSE, PSNR, Contrast, Entropy and Homogeneity have been presented in Table 2 .

Table 2. Performance indices of $D E$ on image fusion.

\begin{tabular}{|c|c|c|c|c|c|}
\hline SET & MSE & PSNR & Entropy & Contrast & Homogeneity \\
\hline 1 & 12447 & 5.4875 & 10.1457 & 0 & 1 \\
\hline 2 & 10253 & 6.0248 & 11.2488 & 0 & 1 \\
\hline 3 & 17305 & 8.1027 & 12.5761 & 0 & 1 \\
\hline
\end{tabular}

\section{RESULTS AND DISCUSSIONS}

MRI and CT images have been fused together using DE. The ultimate objective of image fusion is to acme the valuable data from various input images. The adaptive fuzzy clustering rule has been employed to fragment the region of interest (ROI) to isolate the tumor from the resultant fused image. It will group the various grade intensity segments of the fused image. The segments with huge grade intensity are marked as the tumor, and they have been isolated using thresholding method (Chabira, Skanderi, \& Aichouche, 2013).

Figures 3 (a), (b), (c) and (d) provide the information about the CT and MRI images which are processed for fusion. Figures 3 (a) and (b) displays the gray scale CT and MRI images respectively. Figures 3 (c) and (d) demonstrate the median filtered CT and MRI images respectively. DE-DWT has been involved in the fusing mechanism. Using the fusing rules, fusing rules, the input images have been combined. Diverse levels have been fixed to decide clamor data adversity in the image. Figures 4 (a) and (b) demonstrate decomposed CT and MRI images. Figure 5 illustrates the resultant fused image with decent idiosyncratic enrichment. By following the DE-DST rule, least value of CT is combined with the least level decomposed MRI image to form the fused image. 


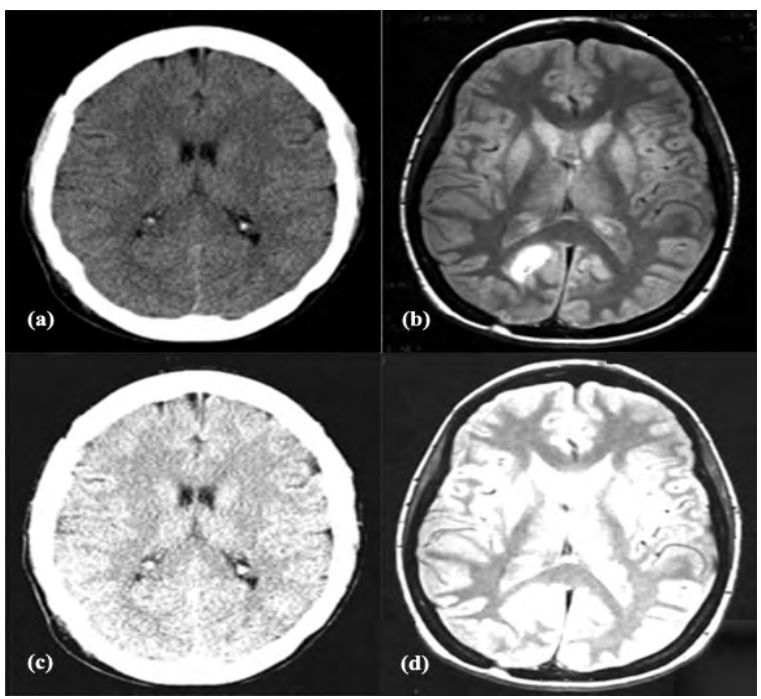

Figure 3. (a) Gray scale CT image (b) Gray scale MRI image (c) Median filtered CT image (d) Median filtered MRI images.

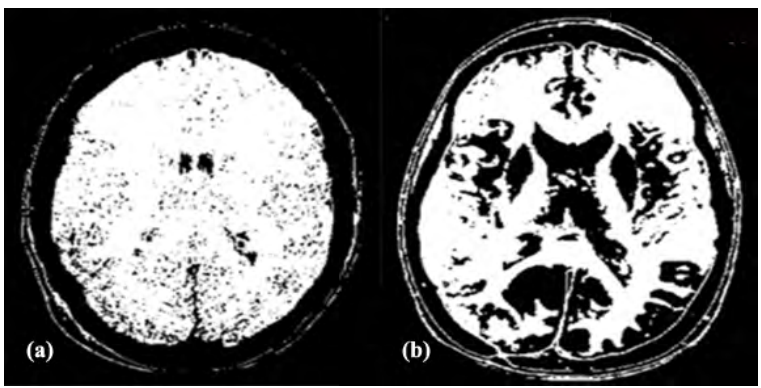

Figure 4. (a) Decomposed CT image (b) Decomposed MRI image.

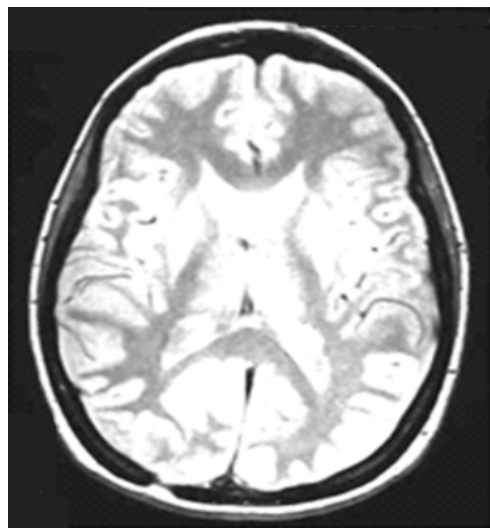

Figure 5. Resultant fused image. 


\section{CONCLUSION}

A typical muscles grown in brain disturb brain activities and that is referred as brain tumor. Biomedical image processing aims to recognize precise data through images with minimum error. Detection of brain tumor via MRI images is not easy due to the intricacy of brain. A pixel based image fusion procedure using DE-DWT has been proposed in this study. The simulations have been carried out with CT and MRI images. The performance indices such as entropy, MSE, PSNR, contrast and homogeneity imply the effectiveness of the proposed DE-DWT approach. 


\section{REFERENCES}

Aslantas, V., \& Toprak, A. N. (2014). Multi focus image fusion by differential evolution algorithm. In Proceedings of the 11th International Conference on Informatics in Control, Automation and Robotics - Volume 2: ICINCO, 312-317, Vienna, Austria. https://doi. $\mathrm{org} / 10.5220 / 0005061103120317$

Chabira, B., Skanderi, T., \& Aichouche, B. A. (2013). Unsupervised change detection from multitemporal multichannel SAR images based on stationary wavelet transform. In 7th International Workshop on the Analysis of Multi-temporal Remote Sensing Images (Multi-Temp). https://doi.org/10.1109/Multi-Temp.2013.6866025

Daneshvar, S., \& Ghassemian, H. (2010). MRI and PET image fusion by combining IHS and retina-inspired models. Information Fusion, 11(2), 114-123. https://doi. org/10.1016/j.inffus.2009.05.003

Ellmauthaler, A., Pagliari, G. L., \& da Silva, E. A. B. (2013). Multiscale Image Fusion Using the Undecimated Wavelet Transform With Spectral Factorization and Nonorthogonal Filter Banks. IEEE Transactions on Image Processing, 22(3), 1005-1017. https://doi.org/10.1109/TIP.2012.2226045

Huang, P. W., Ghen, G. I., Lin, P. L., Ping, G., \& Hsu, L. P. (2014). PET and MRI brain image fusion using wavelet transform with structural information adjustment and spectral information patching. In 2014 IEEE International Symposium on Bioelectronics and Bioinformatics (IEEE ISBB 2014), 1-4. https://doi.org/10.1109/isbb.2014.6820901

Li, Y., \& Liu, G. (2009). Cooperative Fusion of Stationary Wavelet Transform and Nonsubsampled Contourlet for Multifocus Images. In Second International Symposium on Computational Intelligence and Design, 1, 314-317. https://doi.org/10.1109/ ISCID.2009.86

Rowden, A. (2019). Types, symptoms, and treatment of a brain tumor. https://www. medicalnewstoday.com/articles/315625.php

Sahoo, T., \& Patnaik, S. (2008). Cloud Removal from Satellite Images Using Auto Associative Neural Network and Stationary Wevlet Transform. In First International 
Conference on Emerging Trends in Engineering and Technology, 100-105, Nagpur, Maharashtra, India. IEEE. https://doi.org/10.1109/ICETET.2008.99

Shi, H., \& Fang, M. (2007). Multi-focus Color Image Fusion Based on SWT and IHS. In Fourth International Conference on Fuzzy Systems and Knowledge Discovery (FSKD 2007), 2, 461-465. https://doi.org/10.1109/FSKD.2007.414

Singh, R., \& Khare, A. (2014). Fusion of multimodal medical images using Daubechies complex wavelet transform - A multiresolution approach. Information Fusion, 19, 4960. https://doi.org/10.1016/j.inffus.2012.09.005

Wang, L., Li, B., \& Tian, L. F. (2014). Multi-modal medical image fusion using the interscale and intra-scale dependencies between image shift-invariant shearlet coefficients. Information Fusion, 19, 20-28. https:/ / doi.org/10.1016/j.inffus.2012.03.002 
